\title{
Evaluation of Intelligent Transport Systems impact on school transport safety
}

\author{
Dagmara Jankowska-Karpa ${ }^{1, *}$, and Justyna Wacowska-Ślęzak ${ }^{1}$ \\ ${ }^{1}$ Motor Transport Institute, Road Safety Centre, Warsaw, Poland
}

\begin{abstract}
The integrated system of safe transport of children to school using Intelligent Transport Systems was developed and implemented in four locations across Europe under the Safeway2School (SW2S) project, funded by the EU. The SW2S system evaluation included speed measurements and an eye-tracking experiment carried out among drivers who used the school bus route, where selected elements of the system were tested. The subject of the evaluation were the following system elements: pedestrian safety system at the bus stop (Intelligent Bus Stop) and tags for children, Driver Support System, applications for parents' and students' mobile phones, bus stop inventory tool and data server. A new sign designed for buses and bus stops to inform about child transportation/children waiting at the bus stop was added to the system. Training schemes for system users were also provided. The article presents evaluation results of the impact of selected elements of the SW2S system on school transport safety in Poland.
\end{abstract}

\section{Introduction}

The most critical part of the children's route to school and back is the way to/from the bus stop and the area around the stop. It is therefore essential to develop a system that would assist the bus driver, providing them with information about the route, the location of bus stops and the children who should get on/off at the stop. In addition, it is important to improve the visibility of school buses and raise road user awareness by using a uniform sign of school buses in the European Union, which would result in other vehicles reducing their speed or stopping completely when children are boarding or leaving a school bus nearby. The use of Intelligent Transport Systems (ITS) in school transport may also improve the safety of children in traffic. All these elements have been taken into account during the implementation of the Safeway2School - SW2S (Integrated system for safe transport of children to school) European project.

\section{European Project Safeway2School - Integrated System for Safe Transport of Children to School}

The SW2S project was implemented under the 7th Framework Programme of the European Commission. In Poland, it was led by the Road Safety Centre of the Motor Transport Institute. It was coordinated by the Swedish VTI Institute and implemented by fourteen partners from seven European countries.

\footnotetext{
Corresponding author: dagmara.jankowska@its.waw.pl
} 
Under the project, a route planning system, rerouting system and warning system were developed for vehicles in the vicinity of school bus stops. It was also necessary to create a network of intelligent bus stops - IBS (enabling communication between child, stop and bus) and to equip the children participating in pilot studies with special transmitters (tags) that activated bus stop lights.

The target group of the project included primarily school bus drivers, students aged 6 to 16 who use school transport and their families as well as infrastructure managers, vehicle manufacturers, other road users and public administration representatives.

The purpose of the pilot undertaken within the framework of the project was to develop guidelines and recommendations as well as training courses on safe school transport.

Four countries were selected for the pilot implementation of individual Safeway2School system components: Sweden, Austria, Italy and Poland. After the completion of the project, the utility and effectiveness of the technologies employed were evaluated and their acceptance among system users was studied [1].

Figure 1 shows a schematic diagram of the entire Safeway2School system whose components were tested in four pilot locations.

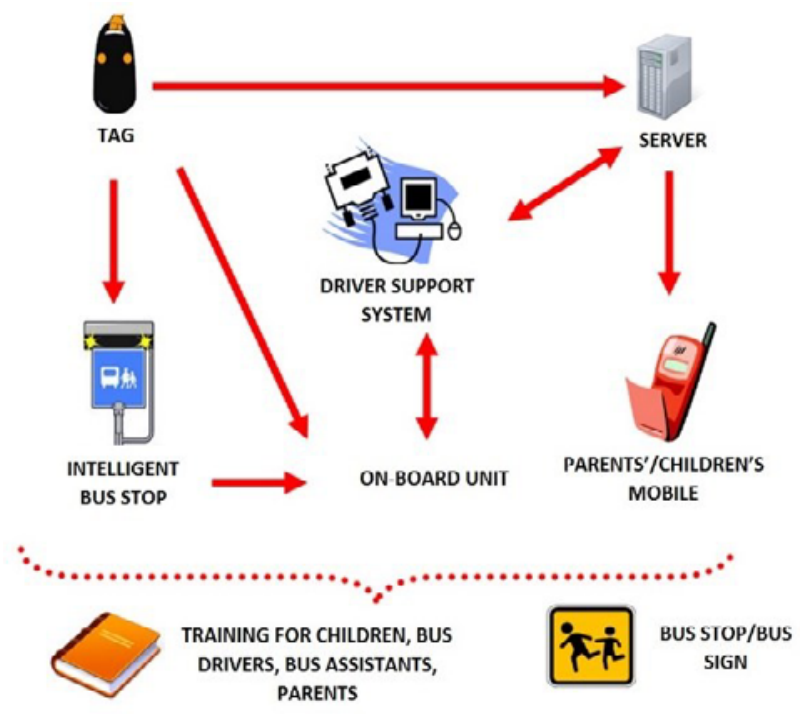

Fig. 1. Diagram of the SW2S integrated system for safe transport of children to school [2].

The Safeway2School system comprises the following components [2]:

- The system for protecting pedestrians at the bus stop and around it - the intelligent bus stop (IBS) and the tag carried by the child. The IBS warning module is powered by solar energy. When the child approaches the stop, his or her tag (using a radio signal) activates the module, which starts flashing its lights.

- The system for assisting the driver interfaces with the bus's on-board computer, using a special application containing information e.g. on the bus route (the bus can be rerouted if unforeseen traffic incidents occur), its speed, stops on the route and the children getting on/off the bus.

- The application installed on the parent's mobile phone, which makes it possible to notify the parent by text message that the child has boarded the bus and has reached his or her school. It is also possible to alert the driver that the child will not be present at the bus stop on a given day. 
- The application installed on the child's mobile phone enables the phone to operate as a transmitter, notifying parents that the child has boarded the bus and has reached school/home.

- Bus stop inventory tool - after inputting data related to e.g. traffic around the bus stop, the speeds of vehicles in its vicinity, the number of people waiting, etc., the safety level of the bus stop can be determined.

- The server which collects all data from the entire system.

The SW2S system is supplemented by specially developed training courses for children, their parents and school bus drivers and also by a special sign developed under the project, which was placed on the school bus and on the stops along its route.

\section{Pilot Implementation of the SW2S System in Poland}

The purpose of the pilot implemented in Poland within the framework of the SW2S project was to study the impact of the new road sign, new school bus sign and IBSs on the behaviour of other road users. The studies focused on drivers of the vehicles that passed the new road sign and school buses with new sign and IBSs. The pilot was carried out in Nowy Dwór Mazowiecki. In the selected primary school, ca. 170 children (out of ca. 500 students attending the school) use the school bus on a daily basis. Among them, 47 were selected for the pilot (in two age groups: aged 7-9 and 10-12) together with their parents and a school bus driver. As part of the pilot, the following SW2S System components were tested in Poland [2]:

- IBSs - 3 stops on the school bus route;

- children's tags - 47 children;

- new bus stop sign - placed on all stops along the school bus route;

- new school bus sign - on the front and rear of the bus.
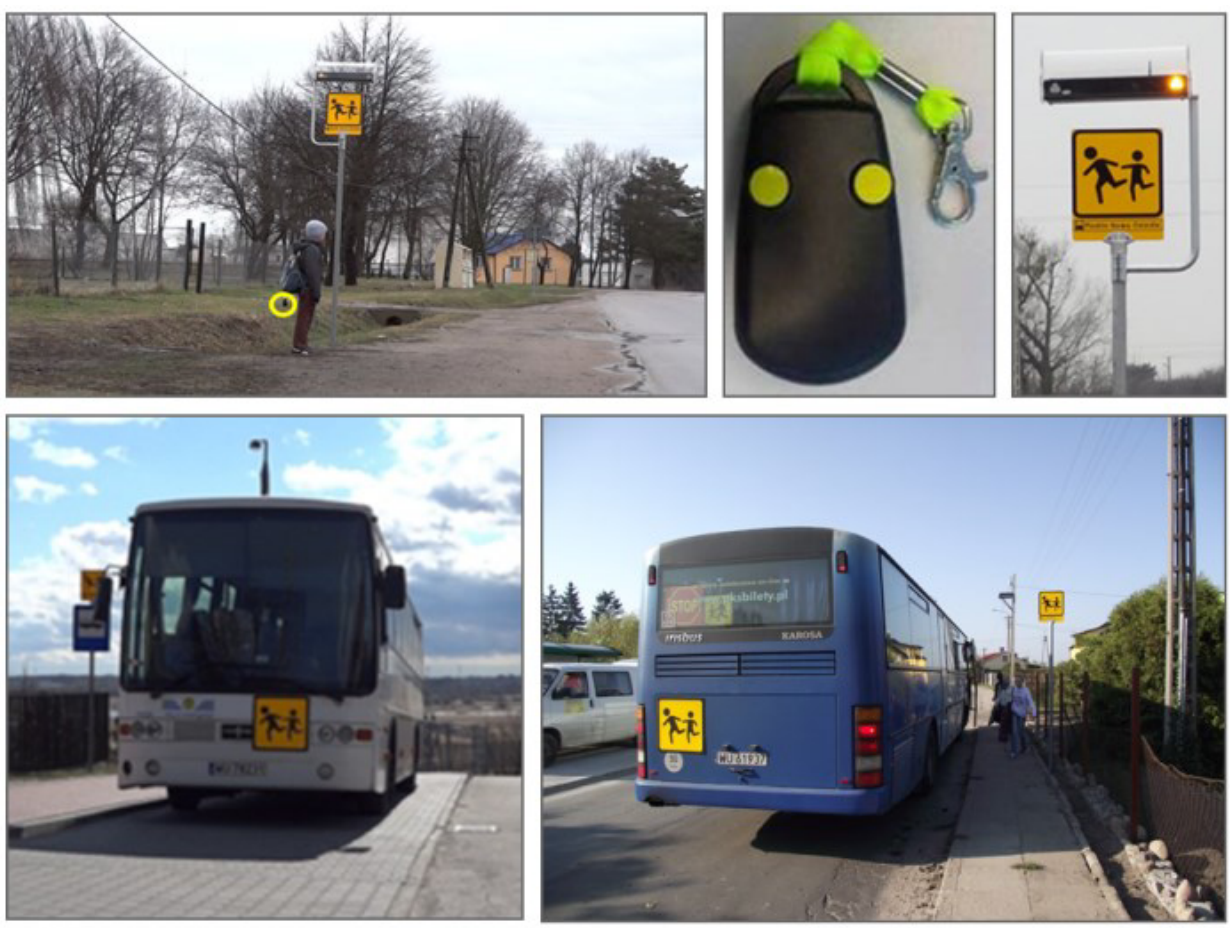

Fig. 2. Elements of the SW2S System tested in Poland [2]. 
The SW2S system was studied in three stages:

- pre-test: before system implementation (vehicle speeds in the vicinity of selected bus stops);

- pre-pilot: installation and testing of system components;

- pilot: system implementation and evaluation.

\section{Impact Assessment of SW2S System}

The following components of the SW2S system were evaluated in Poland:

- impact of new school bus sign:

oparents, children, school bus driver - sense of safety assessment - questionnaires;

o drivers of other vehicles:

- attention and perception assessment - questionnaires;

- tests using eye tracking equipment;

- impact of IBSs on traffic:

othe speed of vehicles passing IBSs (before and after the introduction of the system);

o parents, children, school bus driver - sense of safety assessment - questionnaires;

- training:

o parents, children, school bus driver - sense of safety assessment - questionnaires.

In this article, only the procedure and results of the experiment with the use of eye tracking equipment is presented.

\section{Eyetracking Experiment}

The purpose of the study involving the eye tracking equipment was to assess the effectiveness of IBSs and of new school bus sign in terms of their visibility to motorists driving past the IBSs and past the bus with the new sign [1]. Sixteen subjects participated in the study. The subjects were drivers with category B driving licences. Most of them did not know the road on which they drove during the study. The study involved two buses: one with a new SW2S sign and one with the sign mandated in Poland. The study also covered a bus stop with the IBS module and a stop without this module. Both stops had the new SW2S sign. Each subject encountered the same scenario on the route and the sequence of events was the same in each case.

\subsection{Study Procedure}

The route along which the study was conducted started at stop No. 1 (close to the school) and terminated at stop No. 8 - Wymysły. Travel time amounted to ca. 20 minutes in both directions. A bus with the old sign was stationary at stop No. 7a (waiting for the experimental car) and activated the IBS. The experimental car passed a bus with the new sign, which moved in the opposite direction, close to stop No. 4a. Subsequently, it passed the stationary bus at stop No. 7a (IBS lights were active), passed the last stop (No. 8) and then turned back. When it was passing stop No. 4, the IBS was active and a passenger (pedestrian) was waiting at the stop. Then it passed stop No. 2, which did not include the IBS module but a person was waiting there as well. 


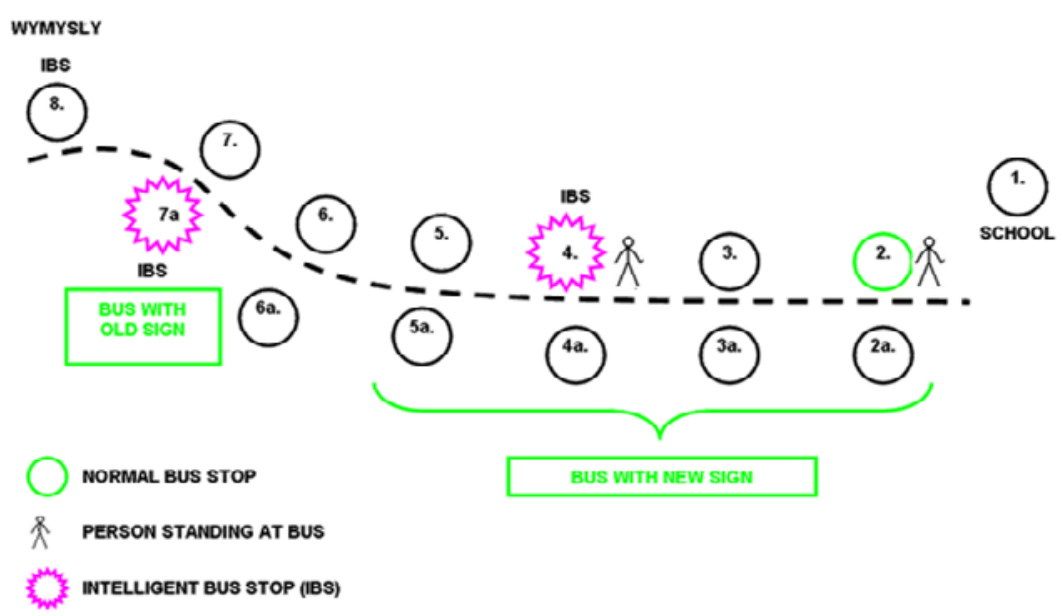

Fig. 3. Scheme of eye tracking experiment route [2].

The study using the eye tracking equipment covered the following situations:

B1: A bus with the new sign follows its normal route from stop No. 1 to stop No. 8 and back, stopping at bus stops. The experimental car passes it in the area of stop No. 4a. B2: A bus with the old sign is stationary at a stop with the IBS module (No. 7a). H1: Children are waiting for the bus at IBS stop - the warning module is active (4). $\mathrm{H} 2$ : Children are waiting at a regular stop (2).

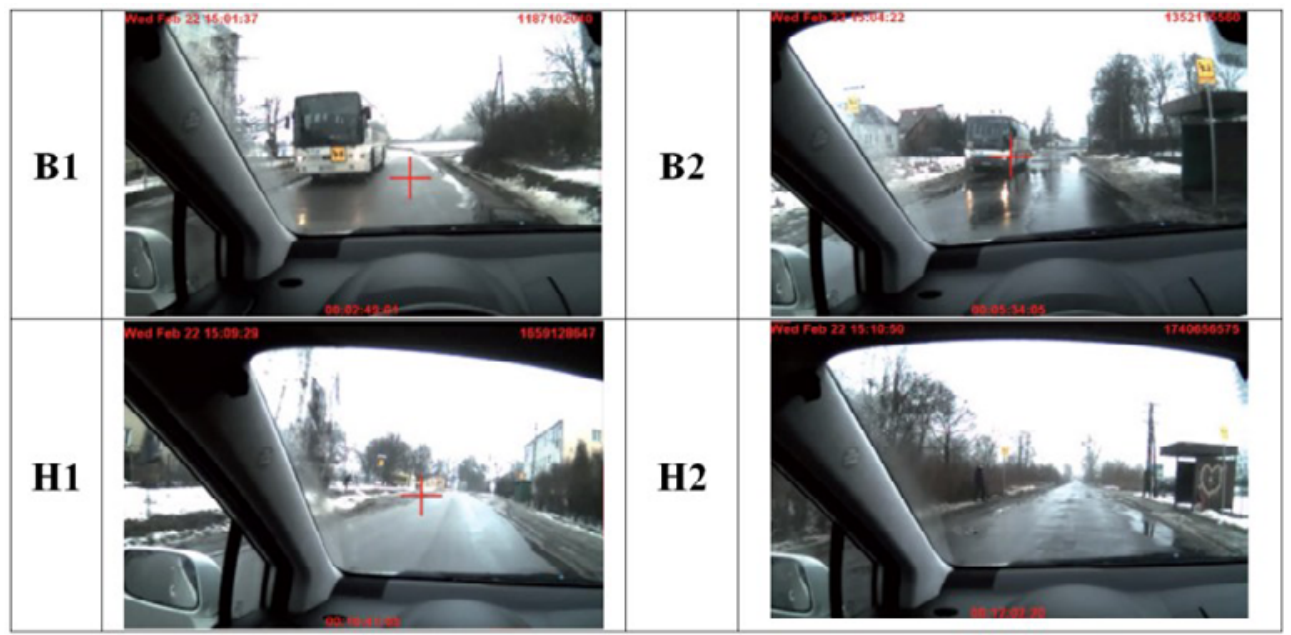

Fig. 4. Situations in the eyetracking experiment [2].

\subsection{Hypotheses}

The hypotheses related to the study were formulated as follows [4]:

- The time during which subjects (experimental car drivers) look at the target (the bus and new signage on the bus) is longer for B1 than for B2 as the new sign is more visible than the one presently mandated. Also, the first look at the new sign occurs earlier than at the old sign. 
- The first look in situation H1 occurs earlier than in situation H2 because the stop with IBS and flashing lights is more visible than a regular bus stop.

- The time during which subjects look at children at IBS (H1) is longer than while looking at children at a regular stop (H2) because IBS is more visible than a regular stop.

\subsection{Study Results}

The numbers in Tab. 1 represent the total time from the first look until the target is passed and the time during which the driver's gaze is fixed on the target in the four analysed situations. Each column in Table 1 represents the mean value for the entire study.

Table 1. Total time from the first look until the target is passed and Time during which driver's gaze is fixed on target (ms) in 4 analysed situations [2].

\begin{tabular}{|c|c|c|}
\hline & $\begin{array}{c}\text { Total time from the first look until the target } \\
\text { is passed (ms) }\end{array}$ & $\begin{array}{c}\text { Time during which driver's gaze is } \\
\text { fixed on target (ms) }\end{array}$ \\
\hline B1 & 17528 & 3161 \\
\hline B2 & 18080 & 4513 \\
\hline H1 & 24320 & 2503 \\
\hline H2 & 19615 & 2020 \\
\hline
\end{tabular}

The total time from the first look until the target is passed is the interval between the time when the subject looks at the target (bus/signage on the bus/children) and the time when the target is passed. This indicator is used because the distance in metres cannot be calculated. The time during which the driver's gaze is fixed is the total time during which the driver looks at the target (bus/signage on the bus/children).

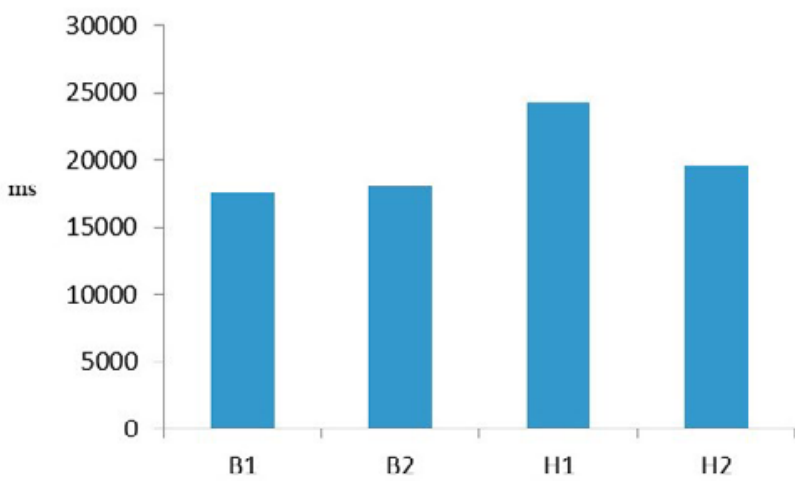

Fig. 5. Total time from first look until the target is passed (ms) [2].

The time of the first look at the target is shorter for situation B1 than for B2. However, in this case the comparison is subject to an error because in situation B1 the bus was moving and, moreover, moving in the direction opposite to the experimental car driver, which reduced the time available. Therefore it is appropriate to assume that the driver spent more time looking at B1 than at B2, which confirms the hypothesis previously adopted.

For situation $\mathrm{H} 1$, the total time from the first look until the target is passed is longer than the time for situation $\mathrm{H} 2$, which also confirms the hypothesis previously adopted, and the 
difference amounts to $4,705 \mathrm{~ms}$. The drivers looked at the bus stop longer when IBS lights were active compared to a stop without the IBS module.

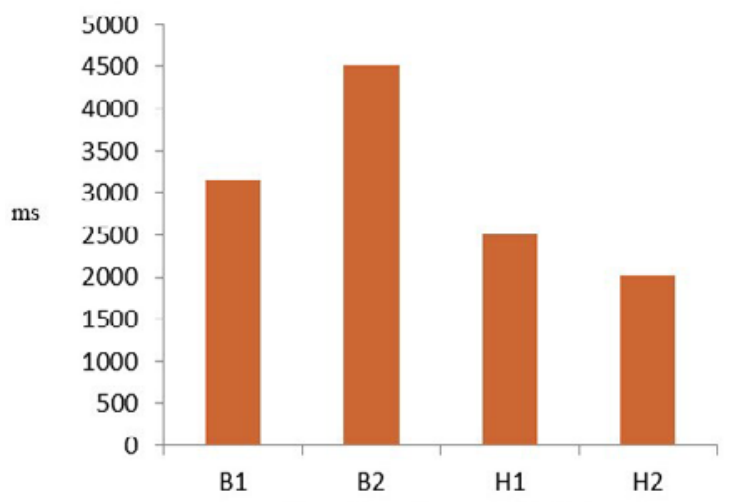

Fig. 6. Time when gaze is fixed on target (ms) [2].

The time during which the driver's gaze was fixed was shorter for situation B1 than for $\mathrm{B} 2$, but - as mentioned above - this was the case because in situation B1, the bus was in motion and moving toward the driver. Comparing situations $\mathrm{H} 1$ and $\mathrm{H} 2$, the time during which the driver's gaze is fixed is longer for H1 (by ca. $483 \mathrm{~ms}$ ), which means that the driver paid more attention to the target (child + IBS) than to the same child waiting at the bus stop without active IBS lights.

Studies on drivers using the eye tracking equipment have successfully confirmed the hypotheses adopted [2].

\section{Conclusions}

The results of the study conducted using the eye tracking equipment indicate that the intelligent bus stop (IBS) attracts more attention from drivers than a regular one. Active bus stop lights cause drivers to pay more attention to what is happening on the road and they notice such stops earlier than regular bus stops without the IBS module.

The results of the study concerning the time when the sign on the bus is noticed and processed are difficult to interpret since the bus with the new sign was in motion. However, slight differences in the total time that elapsed from the first look until the target was passed suggest that drivers noticed the new sign on the bus earlier than the old sign, which also confirms the hypotheses adopted. Additionally, each experimental car driver was asked to complete a short questionnaire after driving the route. The questions concerned, among others, the subjects' knowledge of the speed limits applicable on the route (most drivers knew them, with slight inaccuracies). Most drivers (80\%) paid attention to the new sign on the bus and also to flashing IBS lights (75\%). Most of them also declared that they always slow down when they see people waiting at a bus stop or a bus approaching a bus stop. Two persons declared that they had reduced their speed considerably when seeing flashing lights at the bus stop (IBS).

It should be emphasised that the expected speed reduction of other vehicles on the route was also achieved during IBS activity.

Elements of the Safeway2School system have been evaluated favourably in the studies, which is of considerable importance for ensuring child safety on the route to school and improves road safety. 


\section{References}

1. D. Jankowska, Integrated system for safe transportation of children to school -with the use of Intelligent Transport Systems (ITS), J. KONES Powertrain Transp. 15 (2008)

2. D. Jankowska, J. Wacowska-Ślęzak, Report from pilot implementation of SAFEWAY2SCHOOL system in Poland (Warsaw, 2012)

3. A. Bulling, J.A. Ward, H. Gellersen, G. Tröster, Eye movement analysis for activity recognition, in: Proc. 11th Int. Conf. Ubiquitous Comput. - Ubicomp '09, pp. 41-50 (ACM Press, New York, USA, 2009). doi:10.1145/1620545.1620552

4. A. Bojko, A. Stephenson, It's All in the Eye of the User: How Eye Tracking Can Help Answer Usability Questions, User Exp. 4 (2005) 